\title{
Altered Brainstem Sensory Processing as Assessed by Reflex Modification Procedures in Infants at Risk for Apnea
}

\author{
ENDLA K. ANDAY, MICHELLE E. COHEN, GAIL DAUMIT, AND HOWARD S. HOFFMAN \\ Department of Pediatrics. University of Pennsylvania. School of Medicine, Philadelphia. Pennsylvania 19104 \\ [E.K.A., G.D.]; and The Department of Psychology. The Graduate School of Arts and Sciences, Bryn Mawr \\ College, Bryn Mawr, Pennsylvania 19010 [M.E.C., H.S.H.]
}

\begin{abstract}
Reflex modification procedures were used to test sensory processing in premature infants to examine the relationship between respiratory abnormalities and brainstem neuronal function. A total of 73 premature infants at risk for apnea and/or infants receiving methylxanthine therapy was given a 12-h pneumocardiogram and reflex modification test at a comparable postconceptional age, before discharge. Reflex modification was tested using a controlled eyeblink-eliciting tap to the glabella presented either alone or with a $1 \mathrm{kHz}$ 90-dB SPL tone. The amplitude of the glabellar tap eyeblink and acoustically modified blink were lower in infants discharged on cardiac/apnea monitors $(n=36)$ than in the unmonitored group (1.44 and 1.59 volts versus 2.15 and $2.39 \mathrm{~V}, p<0.005$, respectively). At follow-up, 12 monitored infants had clinically significant apnea after discharge. The records of this subgroup of infants revealed a significantly lower augmentation of the glabellar eyeblink response when compared to all infants screened for respiratory abnormalities and to the other monitored babies $(p<0.01)$. The data suggest that abnormalities of the ventilatory pattern and occurrence of clinical apnea in preterm infants may in some measure be related to acoustic sensory processing, implying an alteration of brainstem neuronal function and organization. (Pediatr Res 26: 576-582, 1989)
\end{abstract}

\section{Abbreviations}

SPL, sound pressure level (re: 0.0002 dynes $/ \mathrm{cm}^{2}$ )

ABR, auditory brainstem response

$M$, monitored infants, $n=36$

$\mathrm{U}$, unmonitored infants, $n=37$

Ap, infants monitored and with apnea following discharge, $n=12$

$\bar{s} A p$, infants screened and without apnea following discharge, $n=61$

Ms̄Ap, infants monitored and without apnea following discharge, $n=24$

HR, heart rate

RM, reflex modification

PNG, pneumocardiogram

PB, periodic breathing

$A_{6} D$, apnea density

LA, longest apnea

TST, total sleep time

REM, rapid eye movement

Received March 30, 1988; accepted July 24, 1989.

Cortespondence Endla K. Anday, M.D., Assistant Professor of Pediatrics, Obstetrics and Gynecology, Pediatric Department, 2 Maloney Building, 36th and Spruce Street, Philadelphia, PA 19104. Supported by NIH Grant HD 10511.
The occurrence of apnea reflects a clinical abnormality with specific etiologies that include sepsis, metabolic disturbances, cardiorespiratory and $\mathrm{CNS}$ disorders, and temperature instability (1). In the absence of various pathologic conditions, apnea may also occur, particularly in the prematurely born infant. Indeed, apnea is the most common disturbance in respiratory control in infants under 28 wk gestational age and in the vast majority of cases resolves by 36 to 37 wk postconceptional age (2). It has been suggested that immaturity of the central nervous system in the premature infant results in a dysfunction of the brainstem respiratory center output, leading to apnea (3). The integration of convergent multimodal input and divergent output is essential for the regulation of respiration. Although the control of respiration draws on many regions of the neuraxis, the reticular formation serves as a major control center for the normal discharge of this information (4). For example, the arousal reaction in ventilatory recovery from hypoxia and hypercapnea, as occur with prolonged apnea, is blunted during sleep in the premature infant (5). An altered arousal response to other stimuli has also been demonstrated in infants exhibiting prolonged apneic events (6).

The hypothesis that immaturity of the central regulation of breathing leads to apnea is supported by the observation that brainstem conduction time, as measured by auditory evoked brainstem response, is prolonged in preterm infants with apnea and decreases as the apneic episodes resolve (7). Although the phenomena may be independent of each other, the anatomical proximity of the regions of the brainstem through which the primary auditory pathway ascends and the areas identified with respiratory neuronal activity may allow for a direct comparison between the integrity of the brainstem auditory pathway and central respiratory function. Current data suggest that respiratory control is effected via the central rhythmic generator, the dorsal and ventral respiratory groups and their afferent input from chemo- and mechano-receptors via spinal cord and cranial nerves IX and $X$ to the tractus solitarius. Rostral inputs from the pons, including the nucleus parabrachialis medialis, Kolliker-Fuse nucleus, locus coeruleus, nucleus of the lateral lemniscus, and the area just caudal to the inferior colliculus also affect the central rhythm generator (8). Although auditory brainstem evoked response testing in infants assesses subcortical maturation of the auditory pathway by detecting a series of volume-conducted neural potentials using electrophysiologic means, it does not provide information of the individual's ability to alter behavior in response to an acoustic stimulus.

The purpose of our study was to investigate the applicability of a psychophysiologic approach based on a behavioral phenomenon called reflex modification to objectively assess sensory processing in a group of high-risk premature infants. The basis for this procedure originated in the observation that a variety of auditory and nonauditory stimuli consistently evoke a startle 
reflex in both humans and animals, the most constant component of which is the eyeblink $(9,10)$. Although the blink is an unconditioned brainstem response, it can be modified by other sensory stimuli via projections from many areas of the nervous system and therefore, is potentially able to reflect processes and mechanisms at different neural levels (11). Investigations in adults using the blink response elicited by a glabellar tap and modified by acoustic signals showed the blink amplitude could be increased (augmented) if the tap accompanied the tone or decreased (inhibited) if the tone preceded the tap by a certain interval (12). Further, modification of the blink reflex could be obtained during both REM and non-REM sleep making this technique ideal for the difficult-to-test subject (13). Although newborn infants do not reliably exhibit inhibition to acoustic signals, they do show augmentation (11). Therefore, we chose to use auditory augmentation of the glabellar eyeblink reflex to examine the relation between CNS sensory processing and clinical apnea in premature infants at risk for apnea.

\section{MATERIALS AND METHODS}

Study Population. Premature infants born at $<36$ wk gestational age as determined by Dubowitz criteria (14) or any infant suffering with recurrent apnea regardless of gestation at birth are candidates for evaluation with cardiorespiratory tracings prior to discharge from the hospital.

A total of 235 babies during an 18-mo period was screened with a 12-h pneumocardiogram; permission to perform reflex modification tests around the time of the pneumocardiogram was obtained on 73 infants who ranged in postconceptional age from 35 to $44 \mathrm{wk}$. Although we obtained permission to perform reflex modification testing in 109 of 235 infants screened with a pneumocardiogram, both tests were not simultaneously performed in 36 infants, thus yielding 73 babies for whom data were available for analysis. At the time of testing, infants were nursed in open cribs, tolerated full feeds, and did not require supplemental oxygen therapy. Evaluation for identification of specific etiologies causing recurrent apnea revealed no pathologic etiology.

Infants identified as demonstrating ventilatory abnormalities on the sleep study (apnea $\geq 20 \mathrm{~s}$ or for $<20 \mathrm{~s}$ when associated with $\mathrm{HR} \leq 80 \mathrm{bpm}$, cyanosis or pallor) $(15)$ or those receiving methylxanthine therapy were discharged from the hospital with a home cardiac/apnea monitor at the discretion of the attending physician and the infant's parents. A total of 36 of 73 infants screened by a pneumocardiogram was discharged on a monitor; 21 of the 36 infants sent home on a monitor were receiving methylxanthine treatment at the time of screening.

At the time of discharge, 41 of the 73 infants tested with reflex modification procedures were evaluated with auditory brainstem response for separate clinical indications. The infants were considered to be at risk for hearing deficits as a result of perinatal complications such as prematurity, asphyxia, and prolonged aminoglycoside therapy. These results were available for comparison with reflex modification testing and sleep study variables. All studies were performed at a time the infant was clinically stable and in preparation for discharge.

RM Procedure. Apparatus. The apparatus for eliciting and detecting eyeblinks has been described previously (16). Briefly, the tap-eliciting device consists of a penlight-size unit that is held to the infant's glabella and by pressing a button on the side of the unit, a miniature solenoid is activated that delivers a controlled tap. Movements of the eyelid are monitored by a lightweight optical detector (Coulbourn module, S76-31, Lehigh Valley, PA) that is positioned in front of the eye and is held in place by the weight of a TDH-39 earphone calibrated with a General Radio Precision Sound Level Meter (model 1561-A, Radio Shack, Fort Worth, TX), fitted with a microphone and a standard type 1 ANSI coupler (re: $2 \mathrm{~cm}^{3}$ coupler calibration). With this arrangement, response amplitude was defined as the peak output of the eyeblink monitoring device during the $150-\mathrm{ms}$ period beginning with the onset of the tap. The output (in V) was sent to a digital voltmeter and stored on disk. The acoustic or tap stimulus was presented in random order as programed and timed by Coulbourn solid-state modules (Coulbourn Instruments: Signal Generators, S81-06; Audio-mixer Amplifiers, S82-24; and Analog-digital Converter, S25-10).

Experimental procedure. The study was reviewed and approved by the University of Pennsylvania Committee on Research Involving Humans. The infants were studied after obtaining parental consent and approximately $30 \mathrm{~min}$ following a feeding while they were asleep. All babies were quiet during the testing with the head turned sideways. The earphone rested lightly over the exposed ear and was held in place by one experimenter. Trials were initiated by placing the tap delivery unit over the glabella; a second experimenter operated the programing apparatus so the first experimenter was unaware of the condition that prevailed on a given trial. Infant state was rated according to behavioral criteria and responses recorded only during active (REM), quiet (non-REM), or indeterminate sleep, or different combinations thereof, were selected for analysis (17).

The procedure for modifying a startle reflex involves variations in a simple two-stimulus paradigm. On control trials, the reflexeliciting stimulus (tap) is presented alone (Fig. 1a). For augmentation trials, a sensory event (tone) is presented simultaneously with the reflex-eliciting stimulus (Fig. $1 b$ ). Specifically, we assessed the ability of an infant to augment the eyeblink response when a $1 \mathrm{kHz} 90 \mathrm{~dB}$ SPL tone with a $2.5 \mathrm{~ms}$ rise/fall time and $50 \mathrm{~ms}$ duration was presented simultaneously with a $16 \mathrm{~V}$ dc tap to the glabella. A blink was readily elicited by this tap intensity in all of the subjects. Each infant received 30 randomly ordered pairs of trials for a total of 60 trials per session that lasted approximately 10 to $15 \mathrm{~min}$. At the end of the session, each subject received five trials of the tone without an accompanying tap to determine if tone alone could elicit a blink reflex; no infant showed a blink response to the tone alone. An infant was considered to have failed the reflex modification test if the mean eyeblink amplitude for trials in which tone accompanied tap was $<10 \%$ above the mean blink amplitude when the tap was presented alone. This criterion was established before any of the reflex modification tests and was based on a review of data we had obtained in previous work with term and preterm infants. Data from our laboratory for term infants has established the within-subject reliability using this technique. Split half, oddeven reliability correlations of 0.92 to 0.95 for individuals $(n=$ 48) during a single testing session were obtained for the glabellar tap and augmented response, respectively. Further, we have found a mean coefficient of variation of $8.5 \%$ within subjects for the glabellar tap within a given testing session and $95 \%$ confidence intervals of 14.7 to $24.8 \%$ for augmentation of the eyeblink in this same group of 48 subjects.

$A B R$. Auditory brainstem response testing was performed in the same room as the reflex modification and carried out without any knowledge of the results of the reflex modification or pneu-

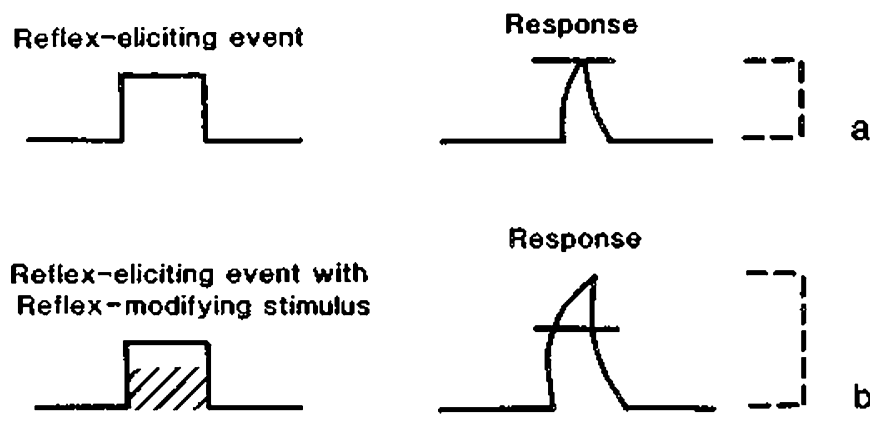

Fig. 1. Schematized representation of reflex modification paradigm. The tap (reflex-eliciting event) results in a blink (reflex response) as is depicted in $a$. The tap accompanied by a tone (reflex-eliciting stimulus) results in an increased blink amplitude (augmentation) as is depicted in $b$. 